\title{
Effects of spanwise spacing on large-scale secondary flows in rough-wall turbulent boundary layers
}

\author{
Christina Vanderwel $^{1}$ and Bharathram Ganapathisubramani ${ }^{1} \dagger$ \\ ${ }^{1}$ Aerodynamics and Flight Mechanics Research Group, University of Southampton, \\ Southampton SO17 1BJ, UK
}

(Received 2 April 2015; revised 14 May 2015; accepted 19 May 2015)

Large-scale secondary flows can sometimes appear in turbulent boundary layers formed over rough surfaces creating low- and high-momentum pathways along the surface (Barros \& Christensen 2014). We experimentally investigate the dependence of these secondary flows on surface/flow conditions by measuring the flows over streamwise strips of roughness with systematically-varied spanwise spacing. We find that the large-scale secondary flows are accentuated when the spacing of the roughness elements is roughly proportional to the boundary layer thickness, and do not appear for cases with finer spacing. Cases with coarser spacings also generate $\delta$-scale secondary flows with tertiary flows in the spaces in between. These results show that the ratio of the spanwise length scale of roughness heterogeneity to the boundary layer thickness is a critical parameter for the occurrence of these secondary motions in turbulent boundary layers over rough walls.

Key words: Turbulent boundary layers, Boundary layer structure

\section{Introduction}

Surface roughness can have a dramatic effect on the dynamics of turbulent boundary layers (Jiménez 2004; Flack \& Schultz 2014). It is typically assumed that the effects of roughness are confined to the roughness layer (Castro 2007; Flack et al. 2007; Amir \& Castro 2011); however, recently it has been observed that large secondary motions in the mean flow can form naturally over rough surfaces and impact even the outer structure of the boundary layer. Mejia-Alvarez \& Christensen (2013) and Barros \& Christensen (2014) observed low- and high-momentum pathways occupied by large secondary swirling motions in the mean flow over the irregular surface topology of a replica of a damaged turbine blade. Reynolds et al. (2007) observed similar secondary flows form over repeating arrays of cubes in a windtunnel and Wang \& Cheng (2006) observed the formation of these secondary flows in open channel flow with longitudinal bedforms consisting of smooth and pointed ridges. Similar structures were also observed by Nugroho et al. (2013) over a surface with converging-diverging riblets, which guided the flow into highmomentum pathways near the wall and in turn created large secondary swirling motions. Numerical simulations by Willingham et al. (2014) of the flows over spanwise-alternating roughness demonstrated that these secondary motions are on the order of the boundary layer thickness and are not particularly sensitive to the roughness height, but have some sensitivity to the width of the roughness elements. The existence of these large secondary

$\dagger$ Email address for correspondence: g.bharath@soton.ac.uk 

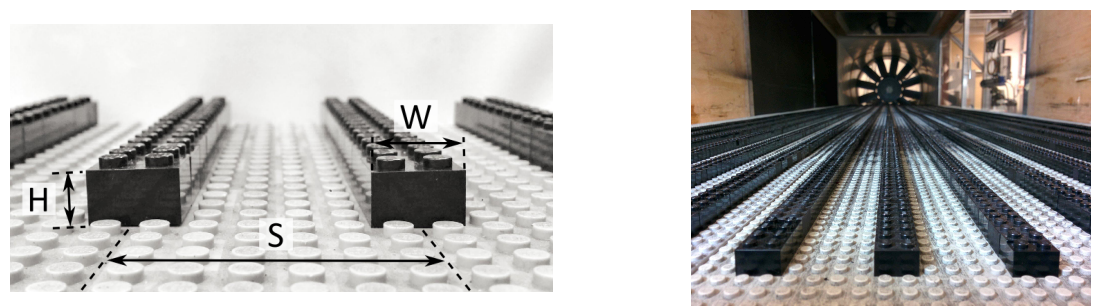

FiguRE 1. Illustrations of the surface topology, with roughness strips aligned in the streamwise direction.

motions clearly illustrates that the effect of the roughness topology on the flow is not necessarily confined to the immediate vicinity of the surface (i.e. the roughness sublayer) but under certain conditions can impact the entire extent of the turbulent boundary layer.

In this study, we intend to specifically investigate the conditions necessary for the formation of large-scale secondary flows by studying their sensitivity to the spanwise spacing of the roughness features. We hypothesize that large secondary vortices only form when the spacing of roughness elements is comparable to the boundary layer thickness. To test this idea, we measure the flow field over five systematically-defined surfaces having streamwise strips of elevated roughness elements with varying spanwise spacing.

\section{Experimental Procedures}

Experiments were performed in the University of Southampton's suction windtunnel using LEGO bricks to form the roughness elements, similar to previous experiments in the same facility (Placidi \& Ganapathisubramani 2015). The wind tunnel has a working section that is $4.5 \mathrm{~m}$ long with a $0.9 \mathrm{~m} \times 0.6 \mathrm{~m}$ cross-section. In this study, strips of LEGO bricks having a width of $W=16 \mathrm{~mm}$ and height of $H=9.6 \mathrm{~mm}$ were aligned with the flow direction and extended over the full $4.5 \mathrm{~m}$ length of the windtunnel test section (see figure 1). These dimensions do not include the array of "bumps" used to connect the pieces together, which have a diameter of $4.8 \mathrm{~mm}$ and a height of $1.7 \mathrm{~mm}$ and which covered uniformly the floor of the windtunnel. The centre-to-centre spacing, $S$, of the elevated strips was varied to test five cases with ratios of $S / W=2,3,6,8$, and 12. We apply the convention that $x, y, z$ are the streamwise, wall-normal, and spanwise directions, $U, V, W$ are the corresponding velocities in those directions. Time-averaged mean values are denoted by an overline, as for example $\bar{U}$, while the fluctuations about the mean are denoted by a small case, as for example $u$, and values that are averaged across the span and in time are denoted by angled brackets, as for example $\langle U\rangle$.

Measurements of the velocity field that developed over the surface were acquired using stereo-PIV in a cross-section normal to the flow direction at a position $4 \mathrm{~m}$ downstream of the leading edge as illustrated in figure 2. This development distance is nearly double the suggested length of $20 \delta$ to ensure fully developed conditions in rough-wall turbulent boundary layers (Antonia \& Luxton 1971; Castro 2007), where $\delta$ is the boundary layer thickness and is reported below in section 3.1. A 200-15PIV Nd:YAG laser by Litron Lasers illuminated the measurement plane and the flow was seeded using a Magnum 1200 fog machine. Particle images were recorded using two LaVision Imager LX 29MP cameras, fitted with lenses having a focal length of $200 \mathrm{~mm}$ and an aperture of f5.6. The cameras were positioned on either side of the measurement plane at angles of approximately $\pm 35^{\circ}$ from the measurement plane and fitted with Scheimpllug adapters 


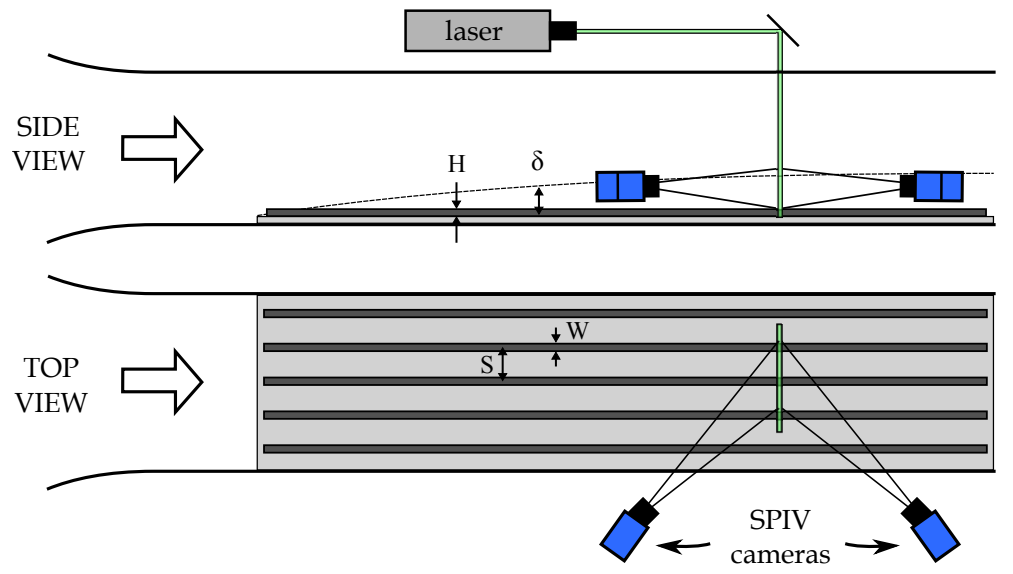

FIgURE 2. Schematic of the experimental setup in the wind-tunnel. Measurements of the velocity field were obtained in a spanwise cross-section of the flow using stereo-PIV.

to accommodate the oblique viewing angle. The coordinate system mapping function for the two cameras was determined using a third-order polynomial fit to images of a double-sided dual-plane calibration target aligned with the measurement plane before the experiment.

In all of the test cases, the freestream velocity was set to $U_{\infty}=15 \mathrm{~m} / \mathrm{s}$. For each case, a total of 1500 image pairs were acquired with an image pair separation time of $15 \mu$ s at a rate of $2 \mathrm{~Hz}$, which was slow enough such that each measurement could be considered independent. Vectors were determined with LaVision's DaVis 8.2.2 software, using window sizes of $32 \times 32$ pixels with $50 \%$ overlap, resulting in a resolution of one vector per $0.9 \mathrm{~mm}$. The instantaneous vector maps were subsequently post-processed in MATLAB to detrend the mean vector maps, which contained a subtle spanwise bias which could have been due to a slight misalignment of the calibration plate with the laser plane. The magnitude of this correction was about $0.2 \mathrm{~m} / \mathrm{s}$, which is on the order of the uncertainty of the PIV measurement and corresponds to approximately $1 \%$ of $U_{\infty}$.

\section{Results}

\subsection{Turbulence properties}

Profiles of the mean streamwise velocity in defect form and the root-mean-square streamwise velocity fluctuations in the boundary layer formed over each of the five surfaces are presented in figure 3. Despite the different spacings of the roughness elements, the $99 \%$ boundary layer thickness for all of the flows is $\delta=108 \pm 1 \mathrm{~mm}$, which results in $\delta / H=11.3 \pm 1 \%$. Although the profiles of the fluctuations appear to collapse across all cases (figure $3 \mathrm{~b}$ ), the mean profiles in figure 3 a diverge into two groups near the wall. We may distinguish the two different groups as those with spanwise spacing much less than the boundary layer thickness, i.e. "fine" spacing, and those surfaces with spacing comparable or larger than $\delta$, i.e. "coarse", as summarised in table 1 . The cases with fine spacing have a larger deficit near the wall, whereas the profiles corresponding to the coarse spacings have fuller profiles. This suggests that coarser spanwise spacings have improved near-wall mixing, bringing in a larger amount of high-momentum fluid to the near-wall region, thereby resulting in a fuller profile.

The skin-friction velocity $\left(U_{\tau}\right)$ was determined from the plateau of the measurements 

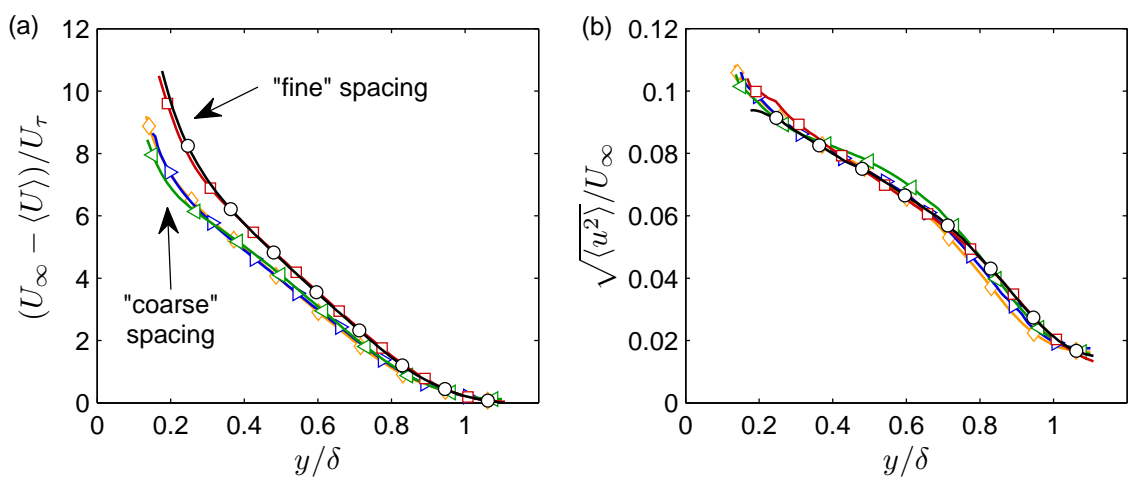

Figure 3. Profiles of (a) the mean velocity deficit and (b) the root-mean-square velocity fluctuations averaged across the span for $S / \delta=0.30(\bigcirc), 0.45(\square), 0.88(\triangleleft), 1.18(\triangleright)$, and $1.76(\diamond)$. Markers are spaced every fifteen data points for clarity.

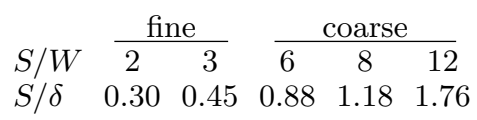

TABLE 1. Summary of the five test cases in the current study.

of the Reynolds stress, $-\overline{u v}$, near the wall (Amir \& Castro 2011). Although the Reynolds stress varies at different spanwise locations, the plateau in the spanwise-averaged stress is consistent between all the different test cases, resulting in $U_{\tau}=0.63 \mathrm{~m} / \mathrm{s} \pm 10 \%$. Maps of the Reynolds stresses are presented later on in figure 6 and the significance of their spanwise variations will be discussed in greater detail below. The Karman number can then be defined as $R e_{\tau}=\delta U_{\tau} / \nu$, and this is in the range $4200 \pm 400$ for all the test cases.

\subsection{Maps of the mean streamwise velocity}

Despite the similarities of the mean profiles, the maps of the mean flow field demonstrate strong spanwise heterogeneities that depend on the surface roughness spacing. These differences are clearly observed in the contour maps of the mean streamwise velocity, which are plotted in figure 4 . For the cases with fine spacing, these spanwise variations are confined to the roughness sublayer $(y<3 \mathrm{H})$, above which the standard deviations of the spanwise fluctuations decrease below $1 \%$ of $U_{\infty}$. In contrast, the surfaces with coarse spacing create spanwise variations that extend to the outer region of the boundary layer. Regions of lower velocity, corresponding to low-momentum pathways, are in the vicinity of the elevated roughness strips, whereas, in general, the unhindered flow away from these regions form high-momentum pathways between the roughness elements. These spanwise variations in the streamwise velocity are most accentuated for the case with surface spacing closest to the boundary layer width $(S / \delta=0.88)$, presented in figure $4 \mathrm{c}$. For this case, the mean velocity exhibits peak-to-peak variations of $18 \%$ at $y / \delta=0.25$ and only decreased to the same level of uniformity as the fine cases for $y / \delta>0.75$. This confirms that the spacing of the roughness is a key parameter in controlling the size and influence of these low- and high-momentum pathways and that by altering nothing but the spacing of the roughness elements, large perturbations to the structure of the mean velocity field may be created. 

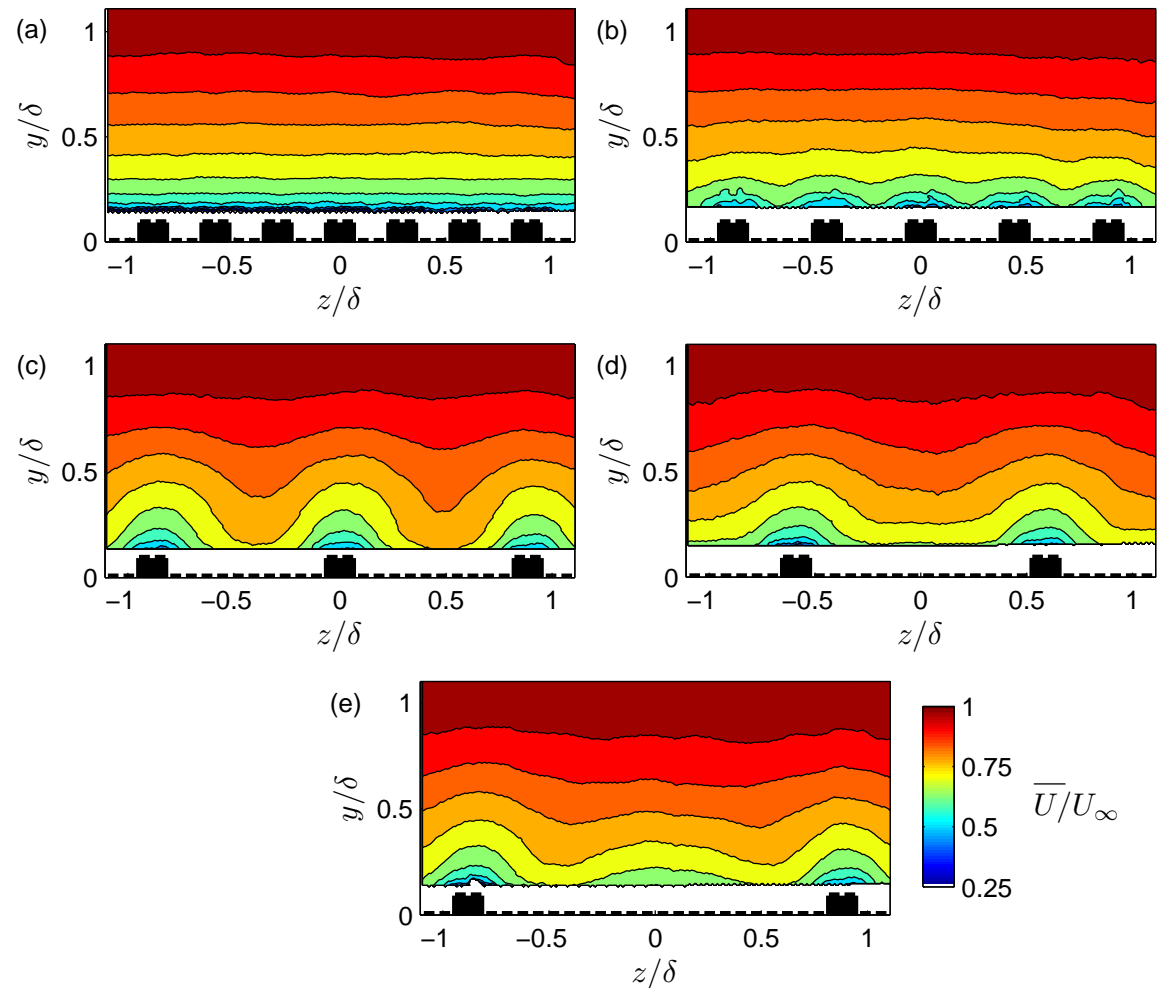

FIGURE 4. Contour maps of the mean streamwise velocity above the roughness elements for the surfaces with $S / \delta=$ (a) 0.30 , (b) 0.45 , (c) 0.88 , (d) 1.18 , and (e) 1.76. The cross-sections of the roughness elements are drawn to scale for reference.

\subsection{Large-scale secondary flows}

The large-scale secondary flows associated with these variations in the streamwise velocity can be easily observed in maps of the mean signed swirling strength and mean in-plane velocity vector fields presented in figure 5. The mean signed swirling strength was calculated as the ensemble average of the instantaneous swirling strength maps, multiplied by the sign of the vorticity as in Anderson et al. (2015), in order to indicate both the strength and direction of rotation of the regions of swirl. For the cases with coarse spacing, large secondary vortices can be clearly observed. The secondary flow consists of a pair of counter-rotating vortices, which are formed on either side of the elevated roughness strips. These are associated with regions of strong upwelling over the elevated regions and wide downdrafts in the valleys. The downdrafts carry with them high velocity from the outer boundary layer and are consistent with the descriptions of high-momentum pathways; similarly, the regions over the elevated roughness correspond with low-momentum pathways. In these measurements, the locations of the low-momentum pathways always occur directly above the elevated roughness elements, which is in contrast to the some previous observations, however, we discuss these differences in section 4.1.

The case with a spacing of $S / \delta=0.45$ is an excellent example of what happens in the limit of the fine-spacing regime (see figure $5 \mathrm{a}$ ). In this case, a small pair of counterrotating vortices (whose sense of rotation is opposite to that of the secondary flows) appear directly above each roughness element, corresponding to a small downwash. These small vortices in fact appear in every case, however, for the coarse spacings, the large 

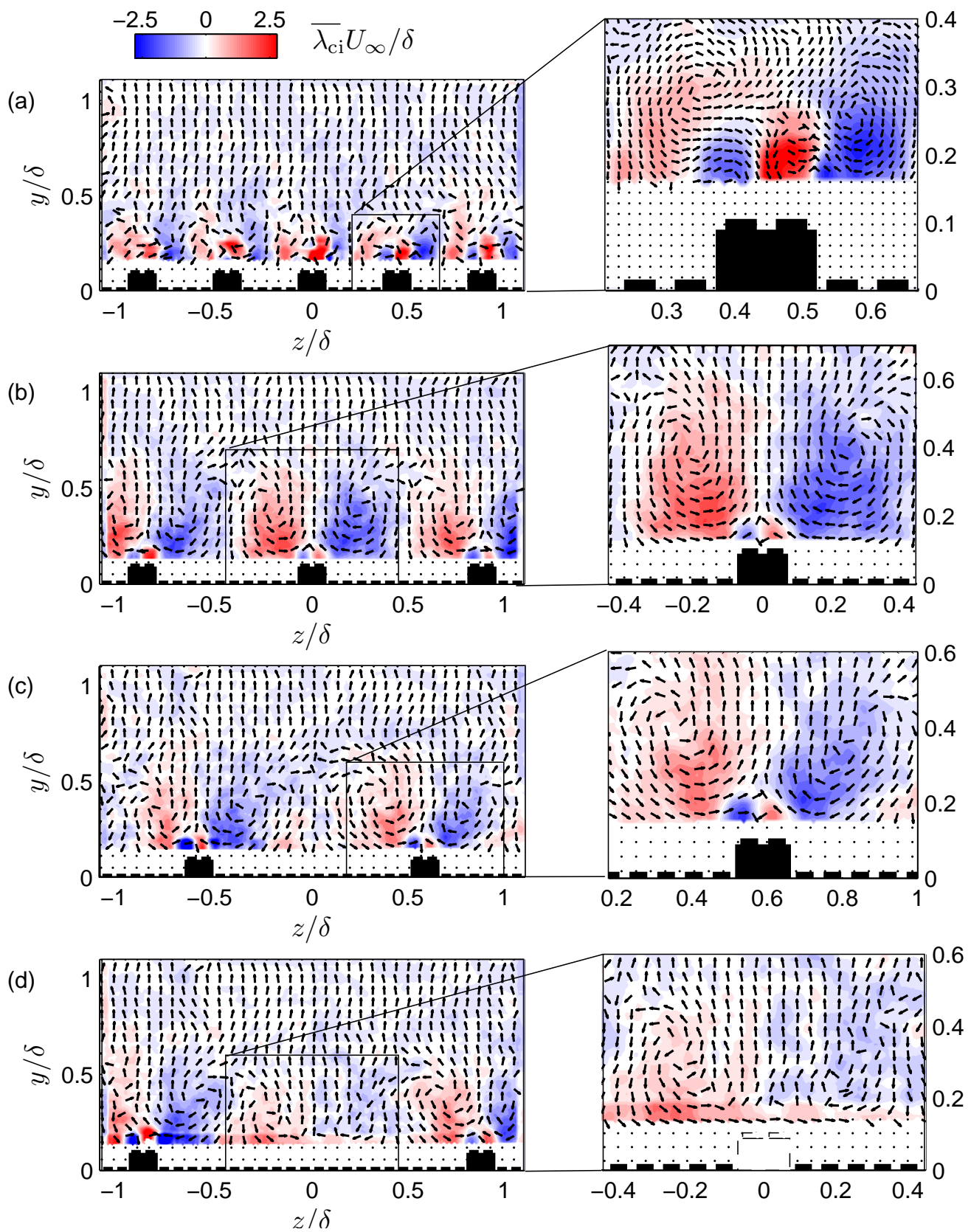

FiguRE 5. Maps of the mean signed swirling strength for $S / \delta=$ (a) 0.45 , (b) 0.88 , (c) 1.18 , and (d) 1.76. The secondary flows that develop over the roughness elements are magnified in (a-c) and in (d) the tertiary flow that forms between the secondary vortices is magnified, where a dashed outline indicates the "virtual" roughness element representing the harmonic corresponding to the wavelength $\sim \delta$. Note that not all vectors are shown in these maps for clarity. 
secondary vortices dwarf these small vortices. If you look closely, similar small vortices may also be observed in the vorticity maps by Anderson et al. (2015). In this limit case with $S / \delta=0.45$, the larger secondary vortices still appear, flanking these smaller vortices; however, the diameter of the larger secondary vortices has nearly the same size as the small vortices that occur above the roughness element, becoming difficult to distinguish in the measurements, and having minimal effect on the mean velocity field. The fact that the sizes of these structures are contained within the roughness sub-layer is consistent with the observation that the spanwise variations in the mean velocity maps are also limited to the roughness sublayer. These structures occurred at even finer scales in the case with the finest surface spacing, which we omitted from figure 5 because it did not exhibit any discernible structure, consistent with the fact that it also did not exhibit large spanwise variations in the mean velocity map.

It appears, therefore, that the size of the secondary vortices is controlled by the roughness spacing, forcing the diameter of each vortex to be approximately one half the roughness spacing. However, this trend stops at the limit when the spacing equals the boundary layer thickness at which point the strength and size of the vortices do not grow any further. The diameter of the large-scale secondary flows was never observed to exceed $\delta$ perhaps because the vertical distance up to which these secondary motions can grow is limited by the boundary layer thickness and therefore so is their spanwise length scale.

In the case when the spacing exceeded the boundary layer thickness $(S / \delta=1.76)$, the flow develops a large-scale tertiary flow, comprised of another pair of counter-rotating vortices (similar to the secondary flow) located at the centres of the valleys between the elevated roughness strips. The strength of these tertiary vortices is roughly half that of the secondary vortices. It appears that as the spanwise spacing increases beyond $\delta$, large-scale tertiary flows start to appear at roughly $\delta$ spacing and presumably will continue to get weaker as the spacing increases further. This observation demonstrates the significance of the boundary layer thickness to the roughness width in the formation of secondary and tertiary flows.

\subsection{Maps of Reynolds stresses}

Representative maps of the normal and turbulent Reynolds stresses that occur in conjunction with these secondary/tertiary flows are presented in figure 6 . We only illustrate the case which exhibit the largest secondary flow $(S / \delta=0.88)$, however, similar patterns were observed in the flows over each of the surfaces with coarse roughness. The patterns of the turbulent Reynolds stresses are similar to those observed in the large eddy simulations (LES) presented by Anderson et al. (2015). The variance of the streamwise velocity $\overline{u u}$ is a maximum in the vicinity of the elevated roughness and particularly near the corners of the roughness elements at the interface of the small vortices and large secondary flows that occur in the low-momentum pathways. The Reynolds shear stress $-\overline{u v}$ is also a maximum near the elevated roughness but exhibits high values that extend nearly to half the boundary layer thickness, following the strong updrafts that occur above the elevated surfaces. Anderson et al. (2015) also observed peaks of $-\overline{u v}$ at the base of the high-momentum pathways (which occurred here in the valleys between the roughness strips); however, we do not capture this region very clearly in our measurements and it is unclear if such a region exists in figure $6 \mathrm{~b}$. The transverse shear stresses $\overline{u w}$ and $\overline{v w}$ are very clearly symmetric and periodic with the roughness spacing, in accordance with the directionality of the mean spanwise velocity $\bar{W}$ imposed by the large secondary flows. Anderson et al. (2015) stressed the importance of the spanwise gradients of the shear stresses and the role they play in driving the secondary vortices, which were interpreted as Prantl's secondary flows of the second kind; the fact that the stresses behave 

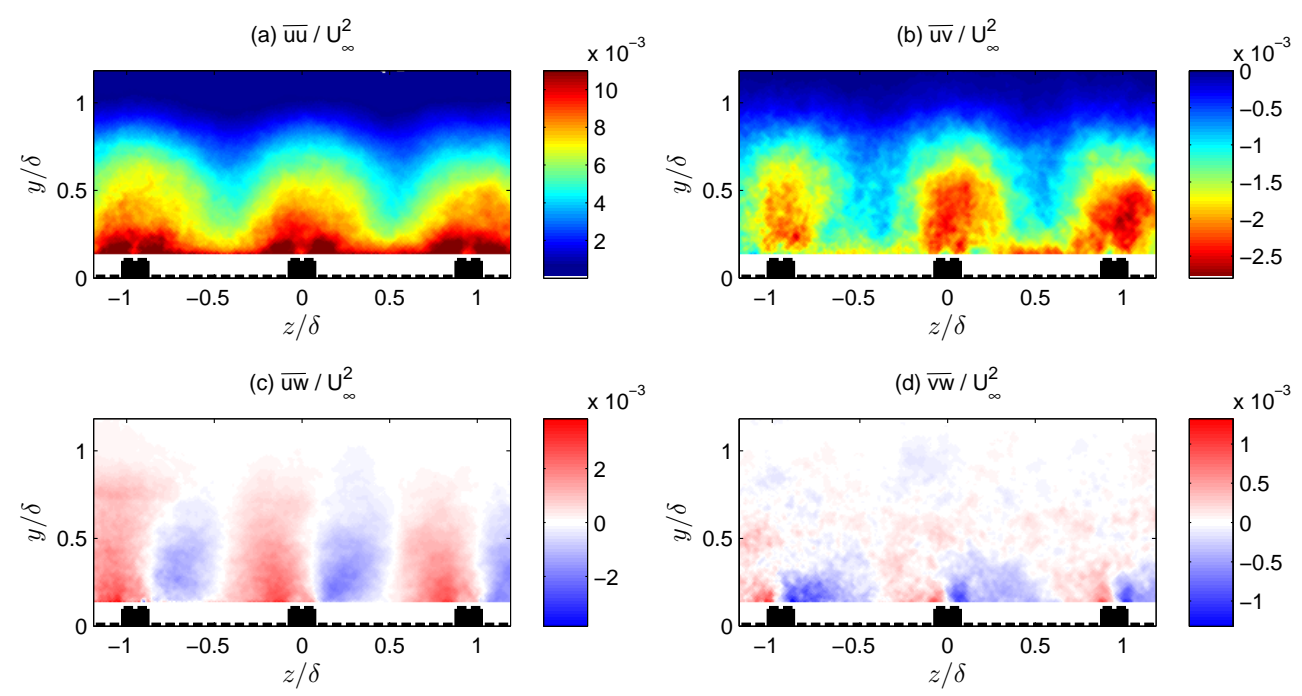

Figure 6. Maps of the turbulent stresses over the roughness spaced by $S / \delta=0.88$, which exhibited the largest secondary flows.

similarly in the current measurements as in their results supports this interpretation. Again, we note that the main difference between these observation and those of Anderson et al. (2015) is the fact that the locations of the low-momentum pathways always occur directly above the elevated roughness elements, whereas in the LES these occurred over what they referred to as 'high' roughness; however, we attempt to explain these differences in section 4.1.

\section{Discussion}

\subsection{On the locations of low- and high-momentum pathways}

In the current measurements, for the cases with coarse spacing where large secondary flows develop, the low-momentum pathways always occur directly above the elevated roughness elements and the high-momentum pathways occur in the valleys between them. This is in contrast to some previous experiments and simulations and at this point we would like to address the reasons for these differences. In the current experiments, the surface topology is constant in the streamwise direction, and the locations of these pathways is constrained by the topology as the flow develops downstream. One could imagine that in the cases with coarse spacing, boundary layers grow over the top and sides of the elevated roughness features, blocking part of the flow area, retarding the local fluid around this blockage, and creating low-momentum zones which persist downstream. In the valleys between the elevated strips, the flow is less inhibited and forms a high-momentum pathway. This is consistent with previous studies over ridged longitudinal bedforms by Wang \& Cheng (2006). So why do the locations differ in some other studies?

In the experimental studies of the flow over the irregular surface of a damaged turbine blade, Barros \& Christensen (2014) (and Anderson et al. 2015) found that low-momentum pathways were systematically positioned above regions with relatively recessed surfaces and they argue that the gradient of the surface elevation could play a role in organising the secondary flows. However, over a three-dimensional surface such as that which was considered in their experiment, boundary layers do not have the opportunity to grow 
significantly over the elevated roughness features before the topology changes. Although the surface appears to have roughness features that are elongated in the streamwise direction, the reality is that there are repeating hills and valleys all along the streamwise direction. The small elevated hills would actually cause a local acceleration of the fluid over them, due to the pressure gradient they impose. This would result in high-momentum pathways generated over elevated roughness features. If the roughness feature persists downstream (instead of forming hills and valleys), the flow would eventually be deviated forming a low-momentum pathway. Therefore, the streamwise continuity of the surface topology is an important factor in the locations of these pathways. One would expect that small gaps in the streamwise continuity of long ridges would not significantly alter the flow as the bulk flow would skim over such gaps. Considering that the separation region behind a cube is known to be roughly $4-5 H$ (Castro \& Robins 1977), one may predict that surfaces with streamwise roughness strips having gaps less than $4-5 H$ would act like continuous strips and exhibit low-momentum pathways coincident with the elevated roughness strips, whereas surfaces with longer gaps would behave more like the irregular surface observed by Barros \& Christensen (2014).

The numerical simulations of Willingham et al. (2014) (and Anderson et al. 2015) considered continuous streamwise-aligned surface roughness and naturally the question becomes, why did this study also observe low-momentum pathways over the 'low' roughness regions? In this case, we attribute this difference to the fact that the simulations do not resolve the roughness topology. The authors reproduce the topology by changing $\Delta U^{+}$(which is the downward shift in the logarithmic region compared with a smooth wall). Their 'high' roughness was simulated by increasing $\Delta U^{+}$, which would have the effect of increasing the velocity gradient at the wall bringing higher momentum flow towards the wall. This would be similar to the flow that would form over a region with recessed roughness in our experiments. Similarly, reducing $\Delta U^{+}$would result in a slower flow similar to that over elevated roughness in our experiments. Therefore, the 'low' roughness regions in the simulations are more akin to the flow created over elevated roughness in the experiments, and likewise 'high' roughness to recessed regions. With this in mind, the current results are consistent with the simulations.

\subsection{Conditions for large-scale secondary flows}

In light of these results, we may speculate that a necessary condition for the formation of these large-scale secondary vortex structures is that the spanwise spacing of the wall roughness is sufficiently coarse $(S / \delta \gtrsim 0.5)$. When the roughness spacing is too fine, any secondary motions do not have the room to grow and are confined to the roughness sublayer. The secondary flows are accentuated the most when the roughness spacing has a period close to the boundary layer thickness; however, larger spacings also generate similar secondary flows but these motions become weaker due to the formation of tertiary flows between the secondary flows. Previous studies by Willingham et al. (2014) have shown that the roughness height does not have a strong effect and that the width of the the strips has only a small influence. Specifically, they tested cases where the ratio of the high and low roughness strips ranged two orders of magnitude from 2-900 and the widths of the strips ranged from $0.2-1.0 \delta$ and found that all the cases produced these types of large secondary motions. Therefore, the spanwise spacing appears to have the most significant effect. We found that all the previous studies we reviewed in the literature that observed large secondary flows do indeed satisfy these requirements. All of the numerical simulations of Willingham et al. (2014) had a spacing of $S / \delta=\pi$. The longitudinal bedforms studied by Wang \& Cheng (2006) were spaced by approximately 
twice the channel depth. Even the converging-diverging riblet-type surface roughness studied by Nugroho et al. (2013) had a spanwise period ranging from $S / \delta=2$ to 2.7 .

The only possible exception that we found in the literature was the experimental study by Reynolds et al. (2007), which observed secondary flows over various repeating surface patterns with periods smaller than the boundary layer thickness. They explained that these structures appeared to be Klebanoff-type modes associated with transition that were organised by the periodicity of the surface roughness. However, they noted that the magnitudes of these secondary flows decreased downstream, as would be expected of transitional phenomena and in contrast to the secondary flows produced by coarsespacings that persist far downstream. Furthermore, they found that when the spacing was finer than $S / \delta \lesssim 0.2$ the secondary flows were no longer detectable. This is consistent with our conclusions and suggests that for short distances the threshold spacing for the development of these secondary flows is reduced in comparison to the far downstream limit where the spacing should be near $\delta$ for large secondary flows to flourish.

For a surface with irregular roughness, a measure of the periodicity can be determined by inspection of the cross-stream autocorrelation function of the surface height. In order to comment on the experimental findings of Barros \& Christensen (2014), we have digitized their surface and calculated the spanwise autocorrelation function. We found that in addition to the initial correlation region extending to approximately $\Delta z / \delta=0.2$, which corresponds to the average width of the roughness features, it also had a local peak at $\Delta z / \delta=0.63$ and 1.18 . These secondary peaks reveal the spanwise spacing of the roughness features, which could be responsible for the development of these large secondary flows. This demonstrates the caution required when designing flows over rough surfaces, because elements with a period near $\delta$ may develop large secondary recirculating regions that disrupt the natural development of the boundary layer.

\section{Conclusions}

This study shows that the presence of large secondary flows is directly related to the spanwise spacing of the surface roughness. These secondary flows consist of large counterrotating recirculation zones that flank the roughness features and are associated with low-and high-momentum pathways. These secondary flows cause large perturbations to the structure of the mean velocity field resulting in significant spanwise deviations that extend beyond the roughness sublayer to over $75 \%$ of the boundary layer thickness.

We determined that a necessary condition for the development and persistance of these secondary vortex structures is that the wall roughness is sufficiently coarse $(S / \delta \gtrsim 0.5)$ and when the roughness spacing is too fine, any spanwise variations are confined to the roughness sublayer. The strength of these secondary vortices appears to be maximized when the spacing is comparable to boundary layer thickness; however, larger spacings also generate secondary flows as well as tertiary flows which together maintain a $\delta$-scale spacing. Because these secondary flows have such a large impact on the structure of the boundary layer and can be created so easily by modifying the surface topology, these flows have important consequences for near-wall mixing, drag reduction, and flow control.

\section{Acknowledgements}

We gratefully acknowledge the financial support from the European Research Council (ERC Grant agreement No. 277472) and EPSRC (Grant Ref No: EP/I037717/1). 


\section{REFERENCES}

Amir, M. \& CAstro, I. P. 2011 Turbulence in rough-wall boundary layers: universality issues. Exp. Fluids 51 (2), 313-326.

Anderson, W., Barros, J. M., Christensen, K. T. \& Awasthi, A. 2015 Numerical and experimental study of mechanisms responsible for turbulent secondary flows in boundary layer flows over spanwise heterogeneous roughness. J. Fluid Mech. 768, 316-347.

Antonia, R. A. \& Luxton, R. E. 1971 The response of a turbulent boundary layer to a step change in surface roughness part 1. smooth to rough. J Fluid Mech. 48 (04), 721-761.

Barros, J. M. \& Christensen, K. T. 2014 Observations of turbulent secondary flows in a rough-wall boundary layer. J. Fluid Mech. 748, R1.

Castro, I. P. 2007 Rough-wall boundary layers: mean flow universality. J. Fluid Mech. 585, $469-485$.

Castro, I. P. \& Robins, A. G. 1977 The flow around a surface-mounted cube in uniform and turbulent streams. J. Fluid Mech. 79 (02), 307-335.

Flack, K. A. \& Schultz, M. P. 2014 Roughness effects on wall-bounded turbulent flows. Phys. Fluids 26 (10), 101305.

Flack, K. A., Schultz, M. P. \& Connelly, J. S. 2007 Examination of a critical roughness height for outer layer similarity. Phys. Fluids 19 (9), 095104.

JimÉnez, J. 2004 Turbulent flows over rough walls. Ann. Rev. Fluid Mech. 36 (1), 173-196.

Mejia-Alvarez, R. \& Christensen, K. T. 2013 Wall-parallel stereo particle-image velocimetry measurements in the roughness sublayer of turbulent flow overlying highly irregular roughness. Phys. Fluids 25 (11), 115109.

Nugroho, B., Hutchins, N. \& Monty, J. P. 2013 Large-scale spanwise periodicity in a turbulent boundary layer induced by highly ordered and directional surface roughness. Int. J. Heat Fluid Flow 41, 90-102.

Placidi, M. \& Ganapathisubramani, B. 2015 Effects of large roughness on aerodynamic parameters and the roughness sublayer in turbulent boundary layers. J. Fluid Mech. (under review).

Reynolds, R. T., Hayden, P., Castro, I. P. \& Robins, A. G. 2007 Spanwise variations in nominally two-dimensional rough-wall boundary layers. Exp. Fluids 42 (2), 311-320.

WANG, Z.-Q. \& Cheng, N.-S. 2006 Time-mean structure of secondary flows in open channel with longitudinal bedforms. Adv. Water Resour. 29 (11), 1634-1649.

Willingham, D., Anderson, W., Christensen, K. T. \& Barros, J. M. 2014 Turbulent boundary layer flow over transverse aerodynamic roughness transitions: Induced mixing and flow characterization. Phys. Fluids 26 (2), 025111. 